\title{
A proposal for a new symbolism to indicate phasors
}

\author{
I. Lombardo \\ Also at DIEEI department of the University of Catania, via S. Sofia, Catania, Italy. \\ INFN - Sezione di Catania, Via S. Sofia, I-95126 Catania, Italy. \\ e-mail: ivano.lombardo@ct.infn.it
}

Received 26 September 2019; accepted 14 October 2019

\begin{abstract}
Even in the most elementary courses on introductory physics at the university level, the use of rotating vectors (or phasors) is of great help to solve problems related to alternating current circuits and to light interference. In this work, I propose a novel notation to indicate a physical quantity that can be represented by a phasor. The symbolism of this new notation appears simple and easily understandable, as testified also by the outcomes of a questionnaire I distributed to students. Furthermore, the results of objective tests made on two samples of students, one trained with and the other one without the new notation, show the potential impact that the new notation can have on the understanding of phasors.
\end{abstract}

Keywords: Phasors; physical education; notation.

PACS: 01.40.-d

\section{Introduction}

It is quite common, in an introductory course on physics at the university level, to deal with physical quantities that vary with the time by following a sinusoidal law. This is observed, e.g., in one-dimension coupled harmonic oscillator problems $[1,2]$, as well as in the calculation of intensity distribution due to light interference in the presence of many slits and in diffraction phenomena [1,3-6]. Certainly, the lion's share is played by the study of electric circuits with alternating currents, a topic that typically covers (alone) an entire chapter of electromagnetism textbooks for science and engineering students, even in the framework of an introductory course [14,7-11].

As discussed by many authors (e.g. [2,6]), when one has to deal with several of such sinusoidal quantities and has to sum many of them, trigonometric calculus can become very long and tedious, and this could divert the attention of the student. For such a reason, many textbooks introduce usually, concurrently with the study of alternating currents, a new physical entity that highly simplifies the calculations: the phasors (or rotating vectors or Fresnel vectors as indicated by some authors, [2]). Depending on the different taste of authors, some textbooks limit the use of phasors primarily to the chapter dealing with circuits in alternating current (e.g. [10]), while others frequently use them also in diffraction studies and to determine the intensity distribution of light when more than two slits are present (see, e.g. [3,4] and[7]). In the last case, the use of phasors represents a very quick and impressive way to understand the appearance of secondary maxima in the intensity distribution [3]. Remarkably that there are educational works in the literature discussing some curious methods to make easy for students the display of phasors and their basic characteristics by using simple materials commonly available (see, e.g., [12-14]).

In more advanced textbooks (see, e.g., [8-10]) the use of phasors is strictly linked to the complex algebra (or symbolic) description of alternating quantities, an expedient that
DOI: https://doi.org/10.31349/RevMexFisE.17.55

greatly simplifies the solution of alternating current circuits problems in which many elements are present, both in series and in parallel connections. The calculation of the complex impedance $\boldsymbol{Z}$ is easily done with the simple rules of complex number algebra, and once determined, it allows us to easily find the relationship between the maximum values of voltage and current, and the difference in phase between the sinusoidal current and voltage. In some text, it is explicitly shown the equivalence between summing two sinusoidal quantities with different phases and amplitudes with the vector sum of two phasors (e.g. [2,6]): I believe this is an important point for making aware of the students of the powerfulness of the phasors approach. Furthermore, following the criteria of Ref. [15], it is easy to recognize that the use of phasor diagrams can aid the process of abduction in students; for example, the interpretation of a phasor diagram in a $R L C$-series circuit can be useful to understand resonance phenomena in $\mathrm{AC}$ circuits. In more advanced courses, it has been suggested in Ref. [16] that phasors can be a very powerful resource to explain the behaviour of resonating systems, without involving a complex mathematical formalism. This point should be taken in high consideration by physics instructors, since the occurrence of resonances is a quite ubiquitous phenomenon in all the branches of physics (see, e.g. [17-21]). The benefits of using phasors to derive elegant demonstrations of theorems in wave optics is beautifully discussed in Ref. [22]; for example, the authors derived simply and straightforwardly the formula of the resolving power for a diffraction grating $(R \equiv \lambda / \Delta \lambda=m N)$.

From the educational point of view, after the work of Worland and Moelter [14], the effectiveness of using phasors for teaching wave optics has been recently probed by Mesic et al [23]. In the latter work, the authors instructed 16-years old high school students about wave optics by using three different approaches: a conventional one, in which the light waves are represented by sinusoidal curves and interference phenomena are seen as "adding multiple curves"; a "sequence of electric vectors (SEV)" approach, using oscil- 
lating electric field vectors for representing light wave, and finally the phasor approach. It is worth noting that the best performances in the understanding of wave optics have been reached by students instructed with the phasor approach [23]. In Ref. [23] it is also pointed out that, even if the use of phasors fulfills almost all the criteria on effective visualization of wave optics phenomena, this approach is currently avoided by several textbooks both at high school or university level. As an example, even in some well-known advanced textbook (e.g. [24]), the symbolic notation is used without explicitly invoking the use of phasors.

Despite their usefulness, phasors are usually indicated with the same symbolism of ordinary vectors, i.e. with bold characters or with an arrow on the top of the physical quantity. This approach, that is formally correct (phasors are vectors, even if their orientation changes continuously with time), could anyway lead to some misunderstanding in students: phasors represent alternating (sinusoidal) quantities with a given angular velocity $\omega$, and they shall not be confused (or mixed) with ordinary three-dimensional vector. This point is briefly discussed on page 261 of Ref. [9].

To avoid this problem, it could be useful to indicate phasors by using symbolism different from the one used to indicate ordinary vectors. Such a procedure is used, for example, in Ref. [9], where an ordinary vector is indicated with a bold roman font, while the complex number indicating the coordinates of the head of a phasor is indicated with a bold sanserif font. However, I believe that the quite similar look of the two notations prevents the identification of a phasor in a given formula at glance; a different choice of notation could, in principle, remove such difficulties. Furthermore, if both phasors and complex numbers are indicated simultaneously with the same notation (e.g., a bold sanserif font), this can lead to difficulties for students to identify the physical quantities varying sinusoidally with the time (and represented by phasors) with respect to complex quantities that are not varying with time, and therefore can not be represented by a phasor (such as the complex impedance).

For completeness, conceptual difficulties arise also by simply using the symbolic method without invoking phasors. As discussed in a footnote in Ref. [11], in this case, it is important to clarify that two different classes of complex numbers must be defined: the "impedance-like" and the "currentlike" ones. The product of an impedance-like complex number and a current-like one has a physical meaning (it would represent a voltage), and also the sum of two impedance-like complex numbers (or two current-like ones) is allowed, but it has no meaning to make the product between two impedancelike complex numbers.

In the next Sections, I illustrate a novel, simple and intuitive notation that I introduced to indicate phasors in a physics course for science and engineering students at the University of Catania, Italy. The main purpose of such a new notation was to mitigate the conceptual difficulties discussed above. In Sec. 2, I will briefly introduce the symbolism and the rationale for adopting such a new notation in physics courses for students of science and engineering; I suggest also a sim-

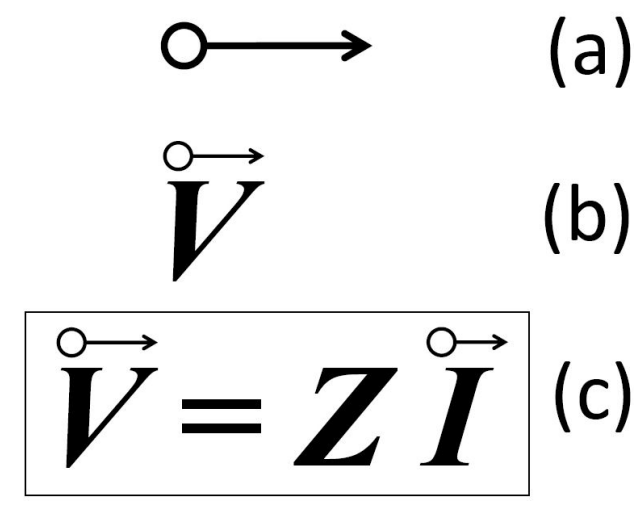

FIGURE 1. A proposed novel symbolism to indicate a phasor. (a) The circle-arrow above-marking that I suggest using when a given physical quantity can be represented a phasor. (b) An example of how the voltage, seen as a phasor, would look in alterative current applications. (c) An example of how the mathematical relation between voltage and current phasors would look, involving the product of a complex number (the complex impedance $\boldsymbol{Z}$ ) with a phasor (the alternating current).

ple typographical way to introduce the new symbolism within the $\mathrm{IT}_{\mathrm{E}} \mathrm{Xenvironment.} \mathrm{In} \mathrm{Sec.} \mathrm{3,} \mathrm{I} \mathrm{will} \mathrm{discuss} \mathrm{the} \mathrm{results} \mathrm{of}$ a preliminary questionnaire I have submitted to students to probe the effectiveness of such a novel notation. Encouraged by the obtained results, I decided to quantitatively evaluate, by using a test, the impact of the new notation in the understanding of the operations with phasors. In Sec. 4, I discuss the preparation phase and the results of such a test; it points out clearly that the new notation has an impact on the understanding of phasors by university-level students.

\section{A new proposed notation for phasors}

Since phasors are rotating vectors, I suggest to use the following symbolism to address a physical quantity that can be represented by a phasor: on top of the letter indicating the physical quantity, I add a symbol made by small circle connected by an arrow, as shown in Fig. 1(a). The look of a phasor representing an alternative voltage should be as in Fig. 1(b). The meaning of the symbolism is quite simple: phasors are vectors (and the arrow in the symbol recalls this property) that rotate with a constant angular velocity around the origin of a bi-dimensional reference frame (and the small circle recalls this property). A relationship between two phasors, e.g. voltage and current in alternating current circuits, would appear as in Fig. 1(c), being $\boldsymbol{Z}$ the complex impedance.

As commonly done in several textbooks (e.g. [8,9]), phasors can be represented by using complex numbers. In fact, by using the Euler's formula, it is quite easy to connect phasors with exponential complex numbers, as shown in Fig. 2: a real number (as $I$ in the case of a current phasor) would indicate the module of the phasor; the angle $\phi$ represents the starting phase of the phasor (i.e. the angle formed by the phasor at $t=0$ with the horizontal, or real, axis), and the $\omega t$ product indicates the variation of the phase angle with the time, responsible for the rotation of the phasor. 


\section{$\stackrel{\leftrightarrow}{\boldsymbol{I}}=\boldsymbol{I} \boldsymbol{e}^{i \varphi} \boldsymbol{e}^{i \omega t}$}

FIGURE 2. The look of an alternating current phasor with the new notation and its symbolic representation with complex exponentials.

I believe that this new notation makes it easier for a student to understand the effect of multiplying a phasor (as a current) by a complex number (as an impedance), avoiding confusion: by indicating the complex number with the exponential form $\boldsymbol{Z}=Z e^{i \alpha}$, the result of the multiplication is a new phasor $\stackrel{\circ}{V}$, with module $Z I$, the same angular velocity of rotation, and a new starting phase, equal to $\phi+\alpha$. In this way, it becomes more difficult for students to make the erroneous statement that every complex quantity (as, e.g., the complex impedance) can be represented by a phasor.

In the environment $\mathrm{AT}_{\mathrm{E}} \mathrm{X}$, it is quite simple to create a symbol with the characteristics here reported. It is sufficient to include the amsmath package and to insert, before the beginning of a document, the following line:

$\backslash$ newcommand $\{\backslash$ phas $\}$ [1] $\{\backslash$ overset $\{\backslash$ mathbin $\{\{\backslash$ circ \mkern-2.5mu\rightarrow $\}\}\}\{\# 1\}\}$

With this user-defined command, it is possible to apply the proposed notation to a given physical quantity (e.g., V) by simply using the command $\backslash$ phas $\{\mathrm{V}\}$ in a mathematic equation. The Equation (c) would, therefore, have the following look:

$$
\stackrel{\circ}{V}=\stackrel{\leftrightarrow}{\longrightarrow}
$$

avoiding any possible confusion between complex quantities (in bold, as usual) and phasors.

\section{Students survey on the new symbolism}

To investigate the impact that the new notation had on a sample of university students, I prepared a simple questionnaire that was submitted to students a few weeks after the end of the course. The questionnaire was composed of 10 questions, with answers going in crescent order $(0=$ in no way, $1=$ few, 2 = sufficiently, 3 = highly, $4=$ very much). The first part of the questionnaire was made by three, more generally, questions asking for the impact that the phasor method has in the understanding of some topics of the course. The second part was made by three questions on the impact that the new notation has in identifying a phasor concerning an ordinary vector or a complex number, and in understanding the result of the product between a phasor and a complex number. The last four questions directly deal with the symbolism of the new notation, and its effect on the understanding of alternating current and optics questions. In the following, I report the list of the ten questions submitted to the students (as translated from the Italian language):
1. How much does, in your opinion, the use of the phasors method simplifies the treatment of phenomena involving physical quantities sinusoidally variable over time?

2. How much is, in your opinion, the use of phasors method useful for the analysis of alternating current circuits?

3. How much is, in your opinion, the use of the phasors method useful for the study of optics phenomena (interference and diffraction)?

4. In your opinion, how much does the new notation used to describe phasors help to distinguish them from ordinary vectors?

5. In your opinion, how much does the new notation used to describe phasors help to distinguish them from complex numbers?

6. Do you think the new notation makes it easier to understand the physical effect that the product of a complex number has on a phasor?

7. How effective is, in your opinion, the new symbolism used to indicate phasors, with respect to its main characteristics (i.e. those of being a rotating vector)?

8. How much has the new symbolism affected your understanding of the functioning of alternating current circuits?

9. How much has the new symbolism used to indicate phasors affected your understanding of interference from three or more slits and diffraction?

10. In your opinion, in which of the two fields of application seen in class (circuits in alternative current and physical optics), would the use of the new notation be more helpful? (Possible options for this question: AC circuits or physical optics).

The number of students answering the questionnaire was $N=25$. In Fig. 3, I show the results of the answers for the questions n. 1-3, 4-6, 7-9. The answers to the $10^{\text {th }}$ question are shown separately in the same Figure. The grouping of questions would reflect their content.

Although this poll is characterized by a limited sample and it may have been (even in part) biased by my style of doing lessons in the classroom, it is possible to draw from it some interesting cause for reflection.

The students consider the use of phasors very important both for the general treatment of quantities sinusoidally varying with time, and specifically for the analysis of circuits in alternating currents: the average scores reached by questions 1 and 2 are very high (3.2 and 3.09 respectively). A slightly lower average score (2.4) and a larger dispersion (root mean square deviation $\mathrm{RMSD}=0.94$ ) are observed for question $\mathrm{n}$. 3 , referring to the use of phasors in optics.

According to the results of questions 4-6, the proposed notation of phasors seems to have had a good impact on students. The average scores of questions 4 and 5 (i.e. on the 

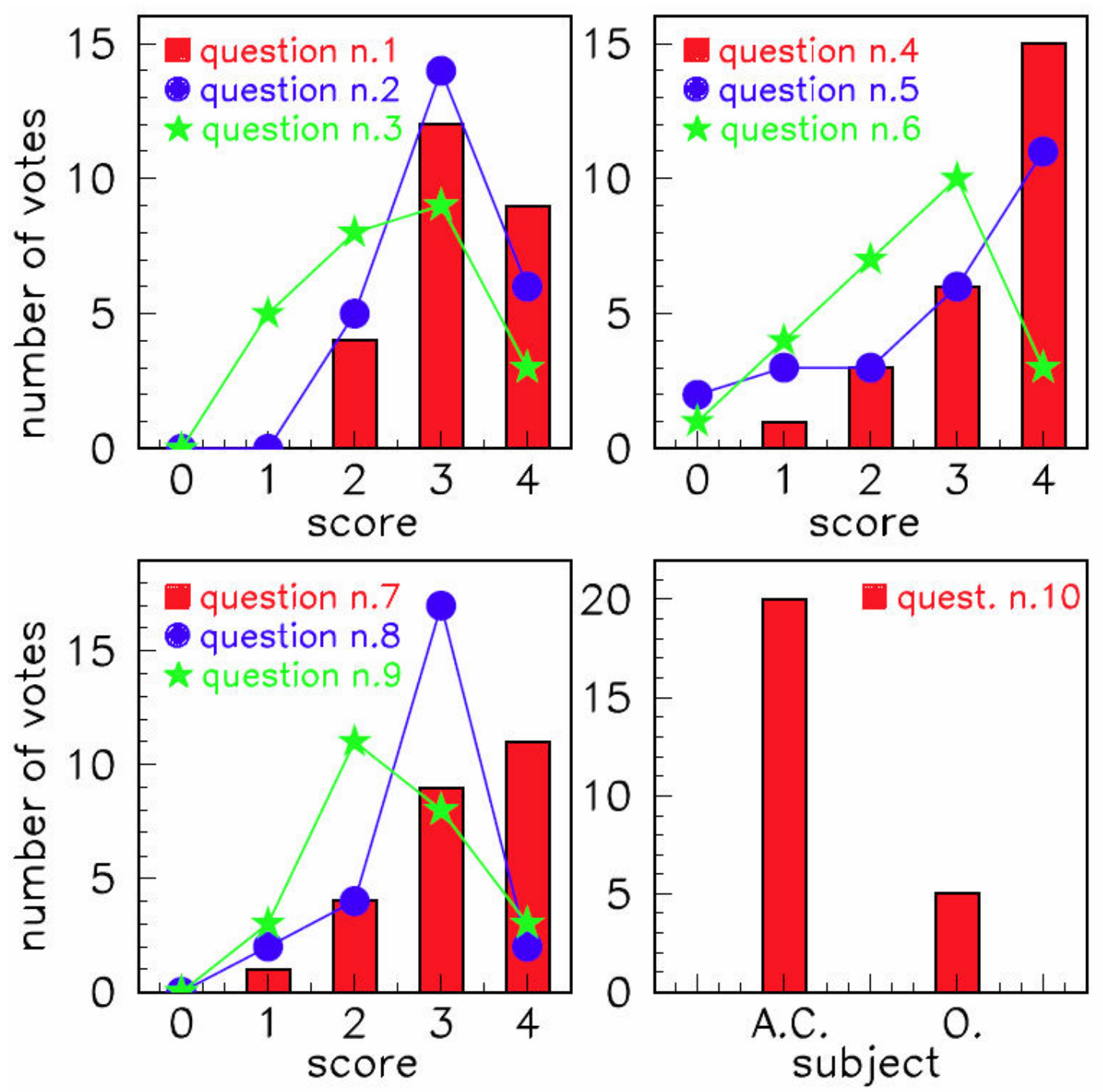

FIGURE 3. Results of the questionnaire submitted to the students of the Experimental Physics II course, for a sample of $N=25$ students. Questions are grouped as indicated by the labels; different symbols and colors indicate the various questions.

impact shown by the new notation in distinguishing phasors from ordinary vectors and complex numbers) are very high (3.4 and 2.84, with the mode equal to the maximum score, 4). A positive impact is seen also for question 6 (mean $=2.4$, mode $=3$ ). The slightly smaller values observed could probably be explained with the intrinsic difficulty for students to deal with a totally new operation ${ }^{i}$, (as the product of a complex number for a phasor) that the new symbolism can only in part alleviate. I believe that an increased ration of exercises and problems in this aspect would help to overcome eventual difficulties on this specific point.

Answers to question 7 (average score 3.2, also in this case with the mode equal to the maximum score, 4) seem to suggest that the new notation has achieved the purpose for which it was conceived: that of iconically representing the main characteristics of a phasor. Answers to question 8 testifies the good impact that the new notation would have on a better comprehension of the functioning of alternating current circuits (average score 2.76, with a small $R M S D=0.71$ ), while the impact of the new notation on a better understanding of optics phenomena seems more limited (average score $2.44, R M S D=0.85$ ), as resulted also from the recorded answers to question 10 .

\section{Impact test on students learning of phasors}

It is not a simple question to investigate if the newly proposed notation has an impact on the effective understanding of the properties of phasors by the students. Nevertheless, I tried to obtain at least some indications by means of a dedicated trial.

First of all, I decided to use a sample of students larger than the previous one to have a statistically significant test. I had the opportunity of using a sample of $N=56$ students following my first-year physics course for the engineering degree in the academic year 2018/19. I split the original sample into two equally populated sub-samples $(N=28)$ based on the first letter of the students' surnames. Then, I prepared two lessons "On the use of phasors in physics" that were identi- 
cal except for the introduction of the new notation in one of the two, and I proposed them to the two sub-samples. I indicate in the following the sample of students having learned phasors with the new notation with the label NN ("new notation"), while the other sample was labeled SN ("standard notation"). It is important to underline that: (1) both the samples of students were totally unaware of the fact that this teaching experiment concerned the study of the impact of the new notation for phasors; (2) before taking part to the test, students had not been taught about phasors at all. Immediately after the lessons, I submitted to the students a free-response test, made by the following questions (translated from Italian):

1. A phasor, with amplitude $4 u$ (with $u$ a given unit of measure) and starting phase $\phi=30^{\circ}$, is multiplied by the complex number $3-4 i$. Which is the result of such an operation?

2. Write, with the symbolic method, the phasor representing a sinusoidally varying quantity $A=5 u \cdot \sin (157 t+$ 26 ), with $u$ a given unit of measure.

3. Which is the relative angle formed by the two phasors representing the following sinusoidally-varying quantities $A=5 u \cdot \sin (20 t+13)$ and $A=7 u \cdot \sin (20 t-6)$, with $u$ a given unit of measure?

4. Which is the relative angle formed by a phasor and its time-derivative?

The evaluation of the free-response test was performed with a simplified docimology: zero points were given to incorrect answers, 0.5 points were given to numerically incomplete (but formally correct) answers, 1 point was given to the right answers. In Fig. 4, I show the average scores reached by the two equal size samples. The red symbols represent the results of the SN sample, the blue ones are the results for the NN sample.

The obtained results are quite interesting. As a first point, I underline that the overall average scores obtained are relatively low (they are under 0.5 for all the questions, except

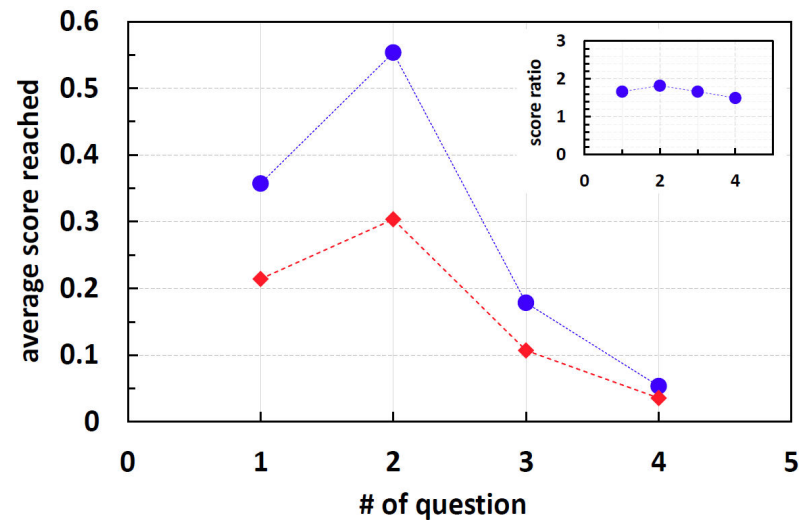

FIGURE 4. Results of the test on the impact of the new notation submitted to students; the average score reached for each question is reported for the NN sample (blue dots) and the $\mathrm{SN}$ sample (red diamonds). The insert shows the ratio between the average scores reached by NN and SN samples.

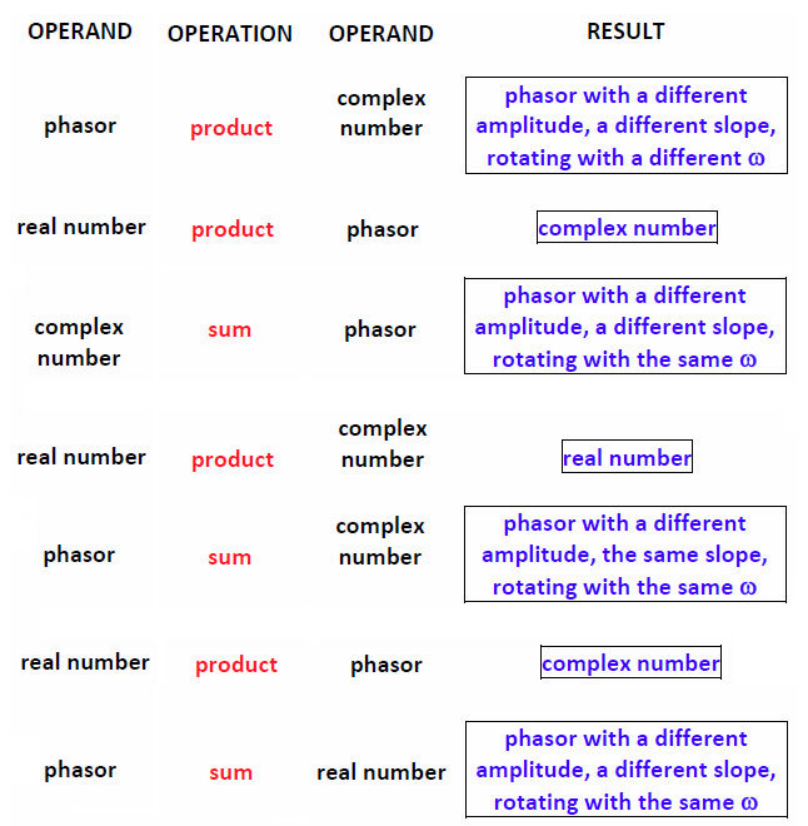

FIGURE 5. The extended matching item test submitted to the two groups of students. All the terms have been translated from Italian.

for the second one for the NN sample). This is not surprising, since we are dealing with a test made immediately after a lesson on a new concept, for which the students have not benefited from a period of individual study. Within this frame, we can also explain the particularly low values for the fourth answer. Students typically encounter more difficulties in applying differential operations to a given physical quantity compared to ordinary operations, as it occurs, for example, with vectors [25].

The interesting point is related to the strikingly higher average scores that the NN sample (blue points) shows compared to the SN (red points) ones. A better visualization of such a finding is shown in the inset of Fig. 4, where I plot the ratio between the NN and the SN average scores. Such ratio is well larger than unity for all the questions (averaging all the questions, it is about 1.6), and it shows a local maximum (of about 1.8) for the second question, i.e., the one concerning the link between the symbolic method and the phasor. Therefore, even in the presence of limited samples of students, it seems that the new proposed notation has a positive impact on the understanding of the main characteristics of phasors, and their operations, that was the main aim of the present work.

Together with the mentioned questions, I asked the students to complete a multiple-columns extended-matching items test, by correctly link the operands, the operators, and the final results shown in Fig. 5 (translated from Italian). Students had thirty minutes to complete the test; during the last five minutes, I allowed them to look at the notes they have taken at the lesson.

The decision to include the extended-matching items test to evaluate the comprehension skills of students is not casual. 


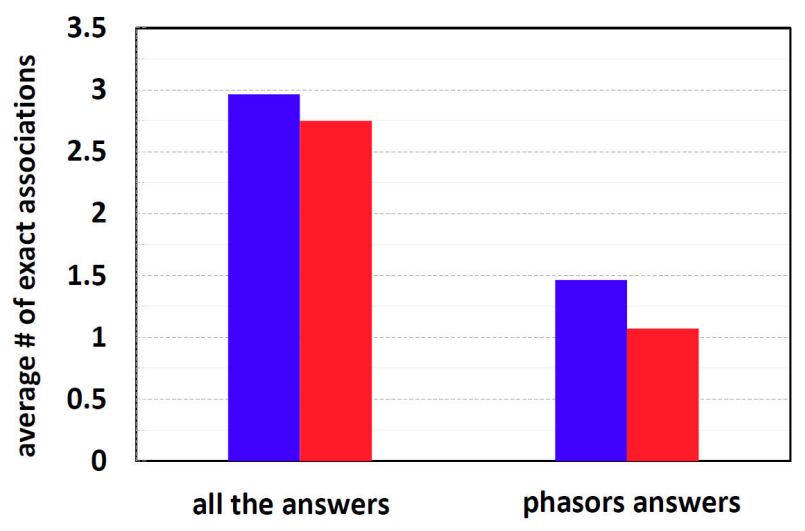

FIGURE 6. Results of the extended matching item test (NN sample in blue and SN sample in red). The outcomes of the test have been divided into two classes: in the first one ("all the answers") I show the results of the whole test, while in the second one ("phasors answers"). I collect the results concerning only the items involving phasors.

As reported in several educational papers in the literature, the matching items test is a promising tool for testing the understanding of concepts just after the completion of a given unit of instruction (see, e.g., [26]). As a second point, the use of extended matching items test (i.e., a matching test with more than five options) has been proven to be a good alternative to free-response questions in examinations where reasoning skills are required (as in medical differential diagnoses, see e.g. [27]). Since the main aim of the new notation was to specialize the concept of phasor at a glance, and since some reasoning effort has to be made to understand the operations between numbers and phasors, both the previous conditions support the choice of the matching items test. In addition to these aspects, the limited time available to perform the test also supports the choice of a matching items test: it has been reported in the literature that, with this type of test, students experience significantly lower test anxiety compared to the multiple-choice formats [28].

The outcomes of the extended-matching items test are summarized in Fig. 6 with the same color scheme used before (NN in blue, $\mathrm{SN}$ in red). Here I report the average numbers of total exact associations (left part of Fig. 6; maximum score $=7$ ), together with the average numbers of exact associations for matching items concerning operations with phasors (right part of Fig. 6; maximum score =4). Also, in this case, the results are quite interesting: the NN sample shows results that are better than the SN sample, in agreement with the results reported in the free-response test. The enhancement of positive results is particularly noticeable for answers concerning operations with phasors. We have, therefore, a further indication of the impact of the new notation on the understanding of phasors and of the operations that can be performed with them.

\section{Conclusions}

In this paper, I propose the introduction of a new symbolism to indicate phasors in the framework of experimental physics courses at the university level. This symbolism pictorially recalls the main aspects of a phasor: its vectorial nature and its rotation around the origin of a bi-dimensional reference frame. According to the results of specific tests submitted to university-level students, the new notation shows a significant potential to reduce the possibility of confusion between phasors and ordinary vectors and between phasors and complex numbers.

From the typographical point of view, the new symbol can be used in any $\mathrm{LT}_{\mathrm{E}} \mathrm{X}$ environment by including the set of line commands discussed in Sec. 2, making it easy to introduce the new notation in new University textbooks.

A first survey on the acceptance of the new notation was performed by submitting a very simple questionnaire to a (still limited) number of students $(N=25)$; the obtained outcomes testify the good impact that it would have both on the understanding of the main properties of a phasor (and this would be at least in qualitative agreement with [23]) and on the approach to the solution of problems involving, in particular, circuits in alternating current.

Starting from these encouraging results, I tried to understand if the proposed new notation had an effective impact on the understanding of phasors by students. To do this, I divided a larger sample of university-level students into two equally populated sub-samples $(N=28)$, the first one trained without using the new notation, the second one with the new notation. Even if the used samples are still limited, the introduction of the new notation shows a statistically significant enhancement in the performance of students trained with the new symbolism on physics topics involving the use of phasors.

According to the stimulating results obtained in this paper, I suggest to the community to make new trials on the use of this notation and to test its usefulness on a much larger sample of students of science and engineering faculties. In this respect, the proposed set of questions and exercises reported in the present paper could be used as a guide-line for new and more extended surveys.

\section{Acknowledgments}

I acknowledge gratefully the students of the University of Catania (Italy) for having allowed me to submit the questionnaire and the test on the novel notation for phasors. Thanks are due to D. Dell'Aquila (Michigan State University, East Lansing, USA) for revising the text and for useful discussions. 
$i$. New in the sense that operations between real numbers, between a real number and a vector, and between vectors have been already discussed in College Physics courses and the Experimental Physics-I course at the university level.

1. M. Alonso and E.J. Finn, Fundamental University Physics, vol. 2: Fields and Waves, 2nd ed. (Addison-Wesley, Reading, USA, 1983).

2. P. Fleury and J.P. Mathieu, Physique génerale et expérimentale, 1st ed. (Eyrolles, Paris, France, 1955).

3. Raymond A. Serway, Physics for Scientist and engineers, 3rd, updated version ed. (Saunders College Publishing, Philadelphia, USA, 1992).

4. D. Halliday, R. Resnick, and K.S. Krane, Physics, 4th ed. (John Wiley \& Sons, New York, USA, 1992).

5. B. Rossi, Optics, 1st ed. (Addison-Wesley, Reading, USA, 1957).

6. F.A. Jenkins and H.E. White, Fundamentals of Optics, 1st ed. (Mc Graw-Hill, New York, USA, 1957).

7. W.E. Gettys, F.J. Keller, and M.J. Skove, Physics: Classical and Modern, 3rd ed. (Mc Graw-Hill, New York, USA, 2007).

8. R.P. Feynman, R.B. Leighton, and M. Sands, The Feynman Lectures on Physics, vols. I and II, 1st ed. (Addison- Wesley, Reading, USA, 1963).

9. I.S. Grant and W.R. Phillips, Electromagnetism, 2nd ed. (John Wiley \& Sons, Chichester, UK, 1990).

10. R. Blum and D.E. Roller, Physics volume two: Electric- ity, Magnetism and Light, 3rd ed. (Holden Day, San Fran- cisco, USA, 1982).

11. E.M. Purcell, Electricity and Magnetism, 2nd ed. (Mc GrawHill, New York, USA, 1965).
12. L. Ribaud and P.G. Mattocks, The Physics Teacher 28 (1990) 160.

13. S. Everall, Physics Education 2 (1967) 49.

14. R.S.Worland and M.J. Moelter, The Physics Teacher 35 (1997) 486.

15. J.J. Clement and M.S. Steinberg, J. Learn. Sci. 11 (2002) 389.

16. Kit Bunker, Physics Education 20 (1985) 281.

17. S. D. Jenkins et al., Phys. Rev. Lett. 116 (2016) 183601.

18. A. Isoya, Nuclear Physics 7 (1958) 126.

19. C. Spitaleri et al., Phys. Rev. C 95 (2017) 035801.

20. I. Lombardo, L. Campajola, E. Rosato, G. Spadaccini, and M. Vigilante, Nuclear Instruments and Methods in Physics Research B 302 (2013) 19.

21. P. L. Connolly et al., Phys. Rev. Lett. 10 (1963) 371.

22. M. Del Mar Sanchez-Lopez, I. Moreno, and A. MartinezGarcia, Education and training in optics and photonics, 1 st ed. (Optical Society of America, Washington DC, USA, 2009).

23. V. Mešić, E. Hajder, K.Neumann, and N. Erceg, Physical Review Physics Education Research 12 (2016) 010135.

24. J.R. Reitz and F.J. Milford, Foundations of electromag- netic theory, 2nd ed. (Addison-Wesley, Reading, USA, 1967).

25. Laurens Bollen, Paul van Kampen, and Mieke De Cock, Physical Review Physics Education Research 11 (2015) 020129.

26. D.W. Zimmerman and R.H. Williams, Psychological Reports 50 (1982) 975.

27. S.M. Case and D.B. Swanson, Teach. Learn. Med. 5 (1993) 107.

28. S.H. Shaha, Edu. Psyc. Meas. 44 (1984) 869. 